\title{
Comments and Corrections
}

\section{Comments on "A Deterministic Approach to the End-to-End Analysis of Packet Flows in Connection Oriented Networks"}

Jean-Yves Le Boudec and Gérard Hébuterne

\begin{abstract}
We prove that the buffer bound in the above paper, can be improved by using a modification of the proofs in the original paper together with so-called network calculus bounds. We also show that the delay bound in the above paper, is the sum of worst-case queueing delays at all nodes along the path of a connection.
\end{abstract}

Index Terms-ATM, delay bound, FIFO, network calculus.

\section{INTRODUCTION}

IN the above paper, ${ }^{1}$ the authors consider a network of discrete time, first in first out (FIFO) queues. They assume that the network uses a connection-oriented paradigm, and that packets (called "cells") all have the same size (as is the case with ATM). In particular, it is assumed that all cells belonging to one connection follow the same path, established at connection setup. In this context, the words "connection" and "flow" have the same meaning. It is further assumed that a connection is spaced at the source by at least the route interference number (RIN) of the connection. The RIN of connection $r$ is defined as the number of occurences of other connections joining the path of $r$. This assumption is called the source rate condition; it is shown in the above paper, that under this set of assumptions:

- network is stable (namely, queue lengths remain bounded);

- delay for any connection is bounded by its RIN;

- maximum buffer required at a queue with $I$ input links and $N_{i}$ connections on input link $i$ is bounded by $\max _{1 \leq i \leq I}\left(N-N_{i}\right)$, with $N=\Sigma_{i=1}^{I} N_{i}$.

In this paper, we show that, under the same assumptions, it is possible to improve the buffer bound to $\min _{1 \leq i \leq I}\left(N-N_{i}\right)$ instead of $\max _{1 \leq i \leq I}\left(N-N_{i}\right)$. This can be achieved using a small variation of network calculus bounds [3], [4], [6], and the above paper, together with a modification of the main proofs in the above paper. This also implies some improvements for the delay bound.

Essentially the same result was found independently by Zhang in [7], using a different approach, based on a detailed analysis of worst case delays. See also some concluding remarks in Section III. In [7], Zhang also analyzes the tightness of the bound.

In Section II, we give the new bounds. The section relies on a number of lemmas, which are given in the Appendix.

Throughout this paper, we adopt a discrete time model, as in the above paper, and assume that all packets have the same size, equal to one unit of data.

Manuscript received May 12, 1999; revised August 17, 1999; recommended by IEEE/ACM TRANSACTIONS ON NETWORKING Editor B. Mukherjee.

The authors are with ICA-EPFL, Lausanne 1015 Switzerland (e-mail: leboudec@epfl.ch).

Publisher Item Identifier S 1063-6692(00)01440-0.

${ }^{1}$ I. Chlamtac, A. Faragó, H. Zhang, and A. Fumagalli, "A deterministic approach to the end-to-end analysis of packet flows in connection oriented networks," IEEE/ACM Trans. Networking, vol. 6, no. 4, pp. 422-431, Aug. 1998.

\section{IMPROVING THE BOUNDS IN [2]}

Our starting point is a number of results, which we collectively refer to as "network calculus" [3], [1], [2], [6]. These results give deterministic bounds on buffer and delay, assuming input processes are limited by some arrival curves, and the service element offers some form of service guarantee. We say that a flow admits a function $\alpha(t)$ as arrival curve if the number of cells that can be observed on the flow during any interval of duration $t$ is $\leq \alpha(t)$. We also say that a node $e$ offers to a flow $r$ a "strict service curve" $\beta(t)$ if, during any time interval of length $t$, for which the backlog of connection $r$ at node $e$ is positive, the number of cells of flow $r$ that are output by the node is $\geq \beta(t)$. The backlog for flow $r$ at node $e$ at some time instant is defined as the number of cells of flow $r$, which have entered node $e$ and did not depart yet. The strict service curve property was defined for example in [5] and is an abstraction of the generalized processor sharing concepts introduced in [5]. The following theorem is a new variant of classical results in [3], [1], [2], and [6].

Theorem 2.1: Consider a node that receives an input connection, with a buffer large enough to avoid discarding data. Assume that the node offers a strict service curve $\beta$ and that the input connection has an arrival curve $\alpha$. Assume that $\alpha\left(u_{0}\right) \leq \beta\left(u_{0}\right)$ for some $u_{0}>0$. Then the maximum buffer occupancy is bounded by $\sup _{0 \leq u<u_{0}}(\alpha(u)-$ $\beta(u))$.

The theorem says that for the computation of a buffer bound, it is sufficient to consider time intervals less than $u_{0}$. The idea is that the busy period duration is less than $u_{0}$.

Proof: The proof is similar to network calculus bounds in [3], [1], [2], and [6]. Call $x(t)$ [respectively, $x^{*}(t)$ ] the input [output] function. This is traditionally defined as the cumulative number of cells observed on the input [output] flow over the time interval $[0, t]$. Consider a given time $t$ at which the buffer is not empty, and call $s$ the last time instant before $t$ at which the buffer was empty. Then, from the strict service curve property, we have

$$
x^{*}(t) \geq x^{*}(s)+\beta(t-s)=x(s)+\beta(t-s) .
$$

Thus the buffer size $b(t)=x(t)-x^{*}(t)$ at time $t$ satisfies

$$
b(t) \leq x(t)-x(s)-\beta(t-s) \leq \alpha(t-s)-\beta(t-s) .
$$

Now if $t-s \geq u_{0}$, then there exists a time $t^{\prime}=s+u_{0}$, with $s+1 \leq$ $t^{\prime} \leq t$ such that $b\left(t^{\prime}\right)=0$. This contradicts the definition of $s$. Thus we can assume that $t-s<u_{0}$.

Now we proceed with a property that generalizes the results inthe above paper and will be required for improving the buffer bound.

Theorem 2.2: With the assumptions in the above paper, consider a given link $e$ and a subset $\mathcal{S}$ of $m$ connections that use that link. Let $n$ be a lower bound on the number of route interferences that any connection in the subset will encounter after this link. Then over any time interval of duration $m+n$, the number of cells belonging to $\mathcal{S}$ that leave link $e$ is bounded by $m$. An equivalent way to formulate the theorem is to say that if we call $\alpha$ the minimum arrival curve for the aggregate of the $m$ flows on link $e$, then we have

$$
\alpha(m+n) \leq m
$$

Before giving the proof, let us mention the following. For $m=1$, the theorem means that the spacing between cell departures from link $e$ is 


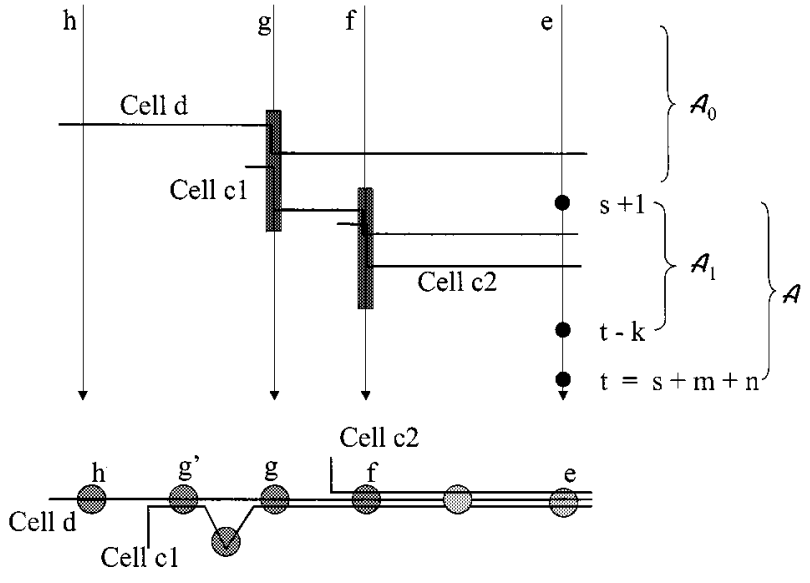

Fig. 1. A time-space diagram illustrating the definitions of $d \preccurlyeq c_{1}$ and $c_{1} \preccurlyeq$ $c_{2}$ (all cells shown are in $\mathcal{S}$ ). Time flows downwards. Rectangles illustrate busy periods. $c_{1}, c_{2}$ are in $\mathcal{A}_{1}$ while $d$ is in $\mathcal{A}_{0}$. The merging point for the flows of $d$ and $c_{1}$ is link $g\left(\right.$ not $\left.g^{\prime}\right)$.

at least $n+1$, where $n$ is the "remaining" route interference number for the connection, past link $e$. In general, $n$ is less than the RIN of the connection. The difference $n-\mathrm{RIN}$ is due to cell delay variation accumulated in the buffers along the path. In other words, a connection gains cell delay variation along its path, and the cell delay variation is bounded by the route interference number consumed along the path. This result (namely, for $m=1$ ) derives immediately from Theorem 1 in the above paper.

However, the theorem is more powerful. It gives a bound on the number of arrivals for an aggregate flow. It can easily be seen that the bound is not a simple consequence of bounds for individual connections; indeed, the bound so obtained would be $\alpha(1+n) \leq m$, instead of $\alpha(m+n) \leq m$. In contrast, the bound in the theorem takes into account global interactions between connections.)

Proof: Remember that we have assumed a connection-oriented network; thus every cell (= packet) belongs to one connection, and every connection uses one path. Consider an arbitrary cell $c$, and call $r$ the connection it belongs to; as a shortcut, we also say that cell $c$ "is in $\mathcal{S}$ " if $r$ is in $\mathcal{S}$. Consider now a fixed time interval $(s, t]=[s+1, t]$ with $t=s+m+n$. Call $\mathcal{A}$ the set of all cells in $\mathcal{S}$ that leave the link during $(s, t]$. Note that connections in $\mathcal{S}$ may interfere at several different links, but since they all end up using link $e$, there is always one last link before or at $e$ at which they interfere. We call this link the merging point of the two connections. We use the classical definition of busy period used in queueing theory, namely, a time interval during which the backlog for the flow at the node is always positive. For two cells $c$ and $d$ in $\mathcal{S}$, and for some link $f$, we say that $d \preccurlyeq c$ if $c$ and $d$ are in the same busy period at the merging points for the connections of $c$ and $d$ (see Fig. 1). We will use the binary relation $\preccurlyeq$ as follows. By Lemma 1 in the above paper, the delay for a cell $c$ in $\mathcal{S}$ due to interferences in $\mathcal{S}$ at the merging point is bounded by the number of cells $d$ in $\mathcal{S}$ satisfying $d \preccurlyeq c$. Our definition of $d \preccurlyeq c$ is very close to the concept of delay chain used in the above paper. More precisely, $d \preccurlyeq c$ at some link $f$ is equivalent to saying that cells $c$ and $d$ are in the same delay chain at link $f$, that link $f$ is the merging point for the connections of $c$ and $d$, and that $d$ reached link $f$ before $c$.

We now define the set $\mathcal{A}_{0}$ as follows; we say that some cell $d$ is in $\mathcal{A}_{0}$ if and only if $d$ is in $\mathcal{S}, d$ leaves link $e$ before or at time $s$, and there exists a sequence of cells $c_{0}=d, c_{1}, \ldots, c_{k}$ all in $\mathcal{S}$, such that $c_{i-1} \preccurlyeq c_{i}$ for $i=1, \ldots, k$, and $c_{k} \in \mathcal{A}$. The definition of $\mathcal{A}_{0}$ is similar to that of the superchain for a given path in the above paper; however, it differs in that it does not apply to one specific path, and that the cells in the chain are restricted to be in $\mathcal{S}$. Call $k=\# \mathcal{A}_{0}$, where the \# sign indicates the number of elements in a set (thus $k$ is the number of connections that are in $\mathcal{A}_{0}$ ). Note that it is possible that $\mathcal{A}_{0}$ is empty, in which case $k=0$. Also call $\mathcal{A}_{1}$ the subset of $\mathcal{A}$ made of those cells that leave the link in $(s, t-k]$. Since the link rate is one cell per time unit, we have

$$
(\# \mathcal{A}) \leq k+\left(\# \mathcal{A}_{1}\right)
$$

The main idea of the proof is that

$$
\left(\# \mathcal{A}_{1}\right) \leq m-k
$$

which together with (2) will prove the result. Equation (3) follows from Lemma A.1, which shows that there can be at most one cell per connection in $\mathcal{A}_{0} \cup \mathcal{A}_{1}$.

We now come to our main result. Consistent with the network model in the above paper, a network node is modeled as a collection of output buffers, with no contention other than at the output buffers. Every buffer is associated with one unidirectional link which it feeds. Every link has one origin node and one end node. We say that a link $f$ is incident to link $e$ if the origin node of link $e$ is the destination node of link $f$. In general, a link has several incident links.

Theorem 2.3: Consider some arbitrary link $e$ with $I$ incident links, and call $N_{i}$ the number of connections that use link $e$ and arrive on the $i$ th incident link, $i \leq I$. Also call $N=\Sigma_{i=1}^{I} N_{i}$. With the assumptions in the above paper, the amount of data in the buffer is bounded by $\min _{1 \leq i \leq I}\left(N-N_{i}\right)$ (instead of $\max _{1 \leq i \leq I}\left(N-N_{i}\right)$ as in the above paper).

Proof: We apply Theorem 2.2 to the set of connections that arrive at $e$ on link $i$, with $m=N_{i}$ and $n=N$. The maximum number of cells that can arrive during $t$ time slots at link $e$ via the $i$ th incident link is thus limited by $\alpha_{i}(t)=\min \left(t, N_{i}\right)$, for $t \leq N$. We now apply Theorem 2.1 to $\alpha=\Sigma_{i=1}^{I} \alpha_{i}$ and the strict service curve $\beta(t)=t$. We can assume without loss of generality that $N_{1} \leq N_{2} \leq \cdots \leq N_{I}$. The function $\alpha-\beta$ is continuous and has a derivative at all points except the $N_{i}$ 's. The derivative changes its sign at $N_{I}\left(=\max _{1<i<I}\left(N_{i}\right)\right)$; thus the maximum of $\alpha-\beta$ is at $N_{I}$ and its value is $N-N_{I}$.

1) The Delay Bound: Last, let us discuss the delay bound. Call $j(r, e)$ the link by which connection $r$ arrives at node $e$. From Theorem 2.3, the delay experienced by a cell of connection $r$ arriving at node $e$ is bounded by

$$
\min _{1 \leq i \leq I(e)}\left(N(e)-N_{i}(e)\right) \leq N(e)-N_{j(r, e)}(e) .
$$

Here we have denoted with $I(e)$ the number of incident links at node $e, N_{i}(e)$ the number of connections arriving at node $e$ on link $i$, and $N(e)=\Sigma_{i=1}^{I(e)} N_{i}(e)$. Now $N(e)-N_{j}(e)$ is the number of route interferences for connection $r$ at node $e$. Also write $e \in r$ to express that node $e$ is on the path of connection $r$. The end-to-end delay for connection $r$ is thus bounded by

$$
\delta(r)=\sum_{e \in r}\left(N(e)-N_{j(r, e)}(e)\right)
$$

which is precisely the RIN of connection $r$. This result is already in the original paper. However, we should mention here first that, contrary to what might be interpreted from the above paper, the end-to-end delay bound is the sum of the local, independent delay bounds at every node. Second, a better bound can be directly obtained by using the left-hand side in (4) instead of the right-hand side. This gives the following bound for the end-to-end delay:

$$
\delta^{\prime}(r)=\sum_{e \text { such that } e \in r}\left\{\min _{i \text { such that } 1 \leq i \leq I(e)}\left(N(e)-N_{i}(e)\right)\right\} .
$$




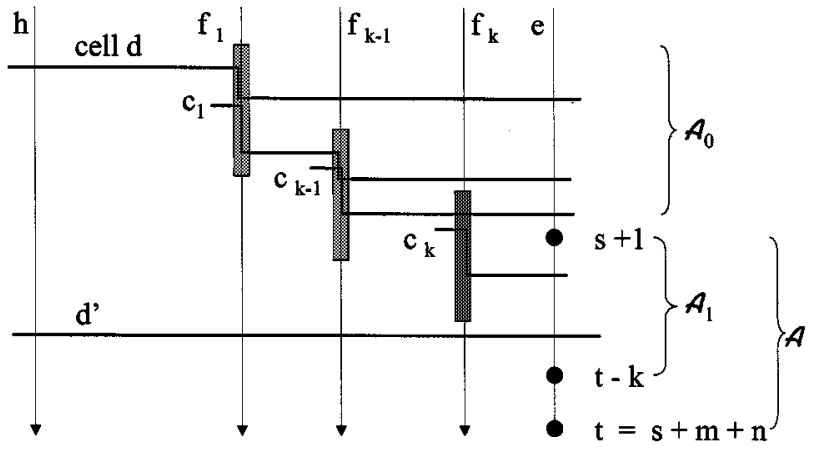

Fig. 2. Two cells of same connection cannot be in $\mathcal{A}_{0}$.

Namely, the end-to-end delay is bounded by the sum of the minimum numbers of route interferences for all connections at all nodes along the path of a connection. For asymmetric cases, this is less than the RIN of the connection as given in (5).

\section{CONCLUDING REMARKS}

Essentially, the same result was found independently by Zhang in [7], using a different approach, not based on network calculus. In our approach, we show an intermediate result (Theorem 2.2), which gives a property of the arrival function for an aggregate number of connections. We believe that this direction could be used to analyze generalizations of the original problem in the above paper, in particular, if we consider more general general source rate conditions.

\section{APPENDIX}

\section{PROOF OF THEOREM 2.2}

We use a discrete time model and assume that all propagation times are zero. The proof in this Appendix can easily be modified to incorporate propagation times, but we prefer to leave this to the reader as this complicates a notation that is already complex enough.

The main technical result is the following lemma. It is an extension of the "excluded superchain" lemma in the above paper.

Lemma A.1: With the notation in Theorem 2.2, there is at most one cell per connection in $\mathcal{A}_{0} \cup \mathcal{A}_{1}$.

Proof: First, we prove that there cannot be two cells of the same connection in $\mathcal{A}_{0}$. We proceed by contradiction. Assume that $d, d^{\prime} \in$ $\mathcal{A}_{0}$ belong to the same connection and were emitted in that order. There exists $c_{0}=d, c_{1}, \ldots, c_{k}$ in $\mathcal{S}$ such that $c_{0} \preccurlyeq c_{1}, c_{1} \preccurlyeq c_{2}, \ldots, c_{k-1} \preccurlyeq$ $c_{k}$, with $c_{k} \in \mathcal{A}_{1}$ (see Fig. 2). Call $f_{i}$ the merging point for the connections of $c_{i-1}$ and $c_{i}$. We show now that cell $d^{\prime}$ must be flowing on the common subpath after cell $c_{k}$. Indeed, otherwise, from the FIFO property, it would have reached $f_{k}$ before $c_{k}$. Assume it has reached $f_{k}$ after $c_{k-1}$; then it would belong to a super chain from $d$ to $d^{\prime}$. This is impossible from Lemma 2 in the above paper. Thus $d^{\prime}$ reaches $f_{k}$ after $c_{k-1}$. By recursion, this shows that $d^{\prime}$ must have reached $f_{1}$ before $c_{0}=d$, which is a contradiction. Thus cell $d^{\prime}$ must be flowing on the common subpath after cell $c_{k}$. Now this contradicts the facts that $d^{\prime} \in \mathcal{A}_{0}$ and $c_{k} \in \mathcal{A}_{1}$.

Second, we show that there cannot be two cells of the same connection in $\mathcal{A}_{1}$. Let $d$ be a cell in $\mathcal{A}_{1}$. Call $s+\tau$, with $\tau \geq 1$ the time at which $d$ leaves the link. From Lemma 1 in the above paper, the delay experienced by $d$ along its path is bounded by the number of interference units experienced by $d$.

Now let us make the distinction between an interference unit, which is due to a connection in $\mathcal{S}$ and which occurs at the merging point with the connection of $d$ (call $r$ the number of such interference units experienced by $c$ ), and other interference units experienced by $d$ (call $r^{\prime}$

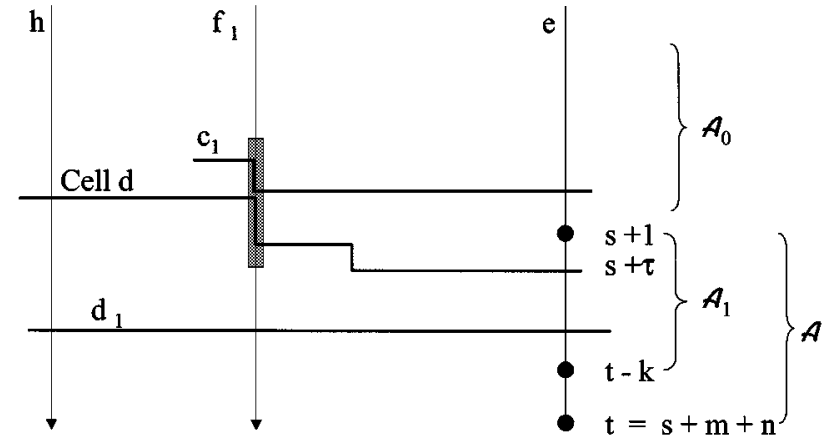

Fig. 3. There cannot be two cells of the same connection in $\mathcal{A}_{1}$.

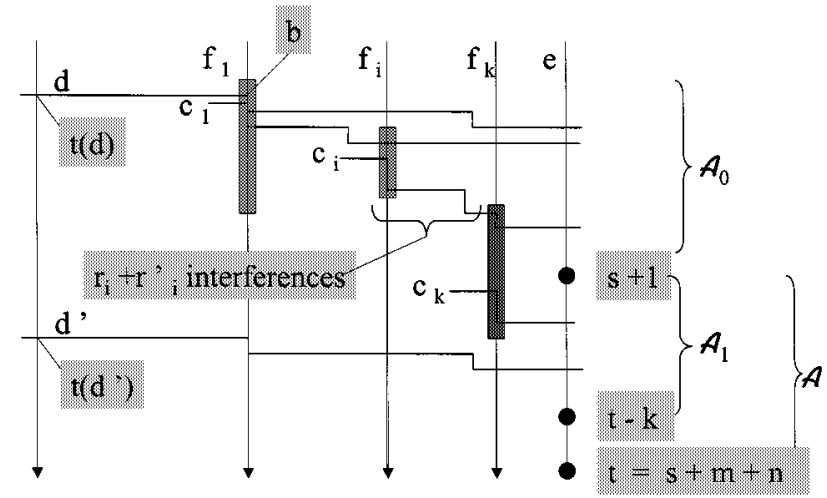

Fig. 4. There cannot be two cells of the same connection in $\mathcal{A}_{0} \cup \mathcal{A}_{1}$.

their number). For example, in Fig. 3, the interference of $c_{1}$ at $g^{\prime}$ is counted in $r^{\prime}$, whereas the interferences of $c_{1}$ at $g$ and $c_{2}$ at $f$ are counted in $r$. The delay experienced by $d$ between its source and link $e$ is thus bounded by $r+r^{\prime}$. Now if $c$ is an interfering cell counted in $r$, we have $c \preccurlyeq d$. Thus either $c \in \mathcal{A}_{0}$ or $c \in \mathcal{A}_{1}$. In the latter case, by the FIFO property, $c$ must leave $e$ before $d$. Thus

$$
r \leq k+\tau-1
$$

Also call $R^{\prime}$ the number of route interferences for the connection of $d$ due to connections that are either not in $\mathcal{S}$ or are in $\mathcal{S}$ but are not at the merging point with the connection of $d$. We have thus $r^{\prime} \leq R^{\prime}$. Call $t_{0}$ the departure time for cell $d$ at its source. From the above we have

$$
t_{0}=s+\tau-r-r^{\prime} \geq s-k+1-R^{\prime} .
$$

Call $t_{1}$ the emission time for the next cell, say, $d_{1}$, following $d$ at its source. By the assumptions in the theorem, the total number of route interferences for the connection of $d$ is at least $(m-1)+R^{\prime}+n$; thus

$$
t_{1} \geq t_{0}+m+R^{\prime}+n .
$$

Combining the two previous equations, we have

$$
t_{1} \geq s+m+n-k+1=t+k+1 .
$$

Thus, from the definition of $\mathcal{A}_{1}, d_{1}$ is not in $\mathcal{A}_{1}$, which proves that there cannot be two cells of the same connection in $\mathcal{A}_{1}$. Third, we prove that if $d \in \mathcal{A}_{0}$ and $d^{\prime} \in \mathcal{A}_{1}$, then necessarily $d$ and $d^{\prime}$ belong to different connections. Consider some $d \in \mathcal{A}_{0}$, and a sequence $c_{0}=$ $d \preccurlyeq c_{1}, c_{1} \preccurlyeq c_{2}, \ldots, c_{k-1} \preccurlyeq c_{k}$, with all cells in $\mathcal{S}$ and $c_{k} \in \mathcal{A}_{1}$. Call $f_{i}$ the merging point for $c_{i-1}$ and $c_{i}$. See Fig. 4. Call $b$ the arrival time of $d$ at $f_{1}$ and $s+\tau$, with $\tau \geq 1$ the departure time for $c_{k}$ at link $e$. Define $r_{i}$ as the number of interference units in the busy period at $f_{i}$ where $c_{i-1}$ and $c_{i}$ interfere, plus those experienced by cell $c_{i}$ between 
$f_{i}$ and $f_{i+1}$, excluding $f_{i+1}$, due to cells in $\mathcal{S}$ at their merging point with the connection of $d$. Following the same reasoning as in the proof of Lemma 2 in the above paper, we have

$$
s+\tau-b \leq \sum_{i=1}^{k}\left(r_{i}+r_{i}^{\prime}\right) .
$$

Call $t(d), t\left(d^{\prime}\right)$ the departure times of cells $d$ and $d^{\prime}$ at their (common) source. We have similarly

$$
b-t(d) \leq r_{0}+r_{0}^{\prime}
$$

where $r_{0}$ [respectively, $r_{0}^{\prime}$ ] is the number of interferences units for cell $d$ on its path from the source to $f_{1}$, excluding $f_{1}$, due to cells in $\mathcal{S}$ at their merging point with the connection of $d$ [respectively, due to other cells].

Now since the spacing between cells at the source is at least the route interference number for the path of $d$ and $d^{\prime}$, we have

$$
t\left(d^{\prime}\right)-t(d) \geq m+n+R^{\prime}
$$

where $R^{\prime}$ is the number of route interferences for the path of $d$, due to connections not in $\mathcal{S}$ at the merging point with the connection of $d$. Thus

$$
\sum_{i=0}^{k} r_{i}^{\prime} \leq R^{\prime}
$$

Combining (7)-(10), we get

$$
t\left(d^{\prime}\right) \geq s+\tau+m+n-\sum_{i=0}^{k} r_{i}
$$

Now every interference counted in $r_{i}$ corresponds to one cell $\gamma$ in $\mathcal{S}$ with $\gamma \preccurlyeq c_{i}$ for some $i$. Thus either $\gamma \in \mathcal{A}_{0}$ or $\gamma \in \mathcal{A}$. By the FIFO property, in the latter case $\gamma$ must leave link $e$ before $c_{k}$. Thus $\sum_{i=0}^{k} r_{i} \leq k+\tau-1$. Thus $t\left(d^{\prime}\right) \geq s+m+n-k+1=t-k+1$ and $d^{\prime}$ is not in $\mathcal{A}_{1}$. Combining the three arguments, we find that there is at most one cell per connection in $\mathcal{A}_{0} \cup \mathcal{A}_{1}$.

\section{REFERENCES}

[1] J.-Y. Le Boudec, "Application of network calculus to guaranteed service networks," IEEE Trans. Inform. Theory, vol. 44, pp. 1087-1096, May 1998.

[2] C. S. Chang, "On deterministic traffic regulation and service guarantee: A systematic approach by filtering," IEEE Trans. Inform. Theory, vol. 44, pp. 1096-1107, Aug. 1998.

[3] R. L. Cruz, "Quality of service guarantees in virtual circuit switched networks," IEEE J. Select. Areas Commun., pp. 1048-1056, Aug. 1995.

[4] A. K. Parekh and R. G. Gallager, "A generalized processor sharing approach to flow control in integrated services networks: The multiple node case," IEEE/ACM Trans. Networking, vol. 2, pp. 137-150, Apr. 1994.

[5] R. L. Cruz and C. M. Okino, "Service guarantees for window flow control," in Proc. 34th Allerton Conf Comm., Cont., and Comp. Monticello, IL, Oct. 1996.

[6] R. Agrawal and R. Rajan, "Performance bounds for guaranteed and adaptive services,", Dec. 1996.

[7] Z. Hongbiao, "A note on deterministic end-to-end delay analysis in connection oriented networks," in Proc. IEEE ICC'99, Vancouver, Canada, 1999, pp. 1223-1227. 